\title{
Prandtl-Batchelor flow past a flat plate with a forward-facing flap
}

\author{
By P. G. SAFFMAN AND S. TANVEER \\ Applied Mathematies, California Institute of 'Texhology
}

(Received 8 August 1983 and in revised form 13 February 1984)

Two-dimensional steady inviscid flow past an inclined flat plate with a forward-facing flap attached to the rear edge is considered for the case when a vortex sheet separates from the leading edge of the flat plate and reattaches at the leading edge of the flap, with uniform vorticity distributed between the vortex sheet and the body. Solutions are found for a particular geometry and a range of values of the vorticity. The method used to calculate the flow is an extension of a free-streamline method widely used in cases where the velocity is a constant on the separating streamline.

\section{Introduction}

Batchelor (1956 b) presented a closed wake model for separated flow of incompressible fluid past bluff bodies in the limit of infinite Reynolds number. This model has inspired a number of attempts to calculate so-called Prandtl-Batchelor inviscid two-dimensional flows past bodies with one or two standing eddies of uniform vorticity separated from the exterior irrotational flow by vortex sheets, because of the possibility that such flows are or may approximate infinite-Reynolds-number limits of Navier-Stokes equations solutions owing to the Prandtl-Batchelor theorem (Batchelor $1956 a$ ) about the uniformity of vorticity in a region with steady closed streamlines in the limit of zero viscosity. However, success to date has been somewhat limited.

Dzugaev (1982) presents an approximate calculation of Prandtl-Batchelor flow past a normal flat plate in a channel based on the assumption that the vortex sheet strength is a constant, but the details are sketchy and it is not clear to us that the solution is consistent. Also, D. I. Pullin (personal communication) reported failure to calculate such a flow past a finite flat plate placed normal to a uniform unbounded stream. Herwig (1982) discusses Prandtl-Batchelor flow over a cavity using asymptotic methods, but arrives at no definite conclusions about the existence of such a flow. The procedure that has been used for numerical calculations is to solve two nonlinear coupled integro-differential equations for the two unknown functions, the vortex-sheet strength and the location of the streamline separating the rotational from the exterior irrotational flow. There are, however, difficulties with such an approach. First, the integrals in the integro-differential equations are singular and there are problems in numerically evaluating them accurately and efficiently. Secondly, as suggested by the results of the free-streamline theory for the case of zero vorticity in the region of closed streamlines, and as confirmed by the results of this paper, the vortex-sheet curvature at the separation and reattachment points is infinite. Failure to obtain solutions does not therefore mean that solutions do not exist and the existence of Prandtl-Batchelor flows in the presence of bodies has remained an important open question in high-Reynolds-number incompressible flows (see e.g. Saffman (1981)). It is worth 
noting that there have been successful calculations of Prandtl-Batchelor flows in the absence of physical bodies. For instance, Sadovskii (1971) considers vortex regions in a potential stream with a vortex sheet on the boundary and finds a one-parameter family of solutions for different jumps of the Bernoulli constant across the vortex sheet. Deem \& Zabusky (1978) and Pierrehumbert (1980) have calculated steady motion past pairs of uniform vortex regions of equal and opposite vorticity with no vortex sheet imbedded in an irrotational flow.

In this paper, we demonstrate numerically the existence of a Prandtl-Batchelor flow for a particular geometry of some aerodynamic interest. The calculation is done for two-dimensional inviscid flow past a flat plate with a forward-facing flap attached to the rear edge (figure 1). The separating streamline and hence the vortex sheet goes from $A$ to $B$, dividing the fluid-flow region into two: an outer irrotational part (region I) and an inner inviscid but rotational part (region II) with vorticity $\omega$. This geometry is of interest in achieving high lift on an airfoil, and has been investigated experimentally and calculated theoretically for the case $\omega=0$ (Hurley 1959) using Kirchhoff-Helmholtz free-streamline theory. Our method of calculation is very different from the integral-equation approach, and is an extension of the functiontheoretic approach of complex variables which has been used successfully in problems where the velocity on the separating streamline is a constant. Our numerical evidence suggests strongly that Prandtl-Batchelor flows indeed exist, at least for asymmetrical geometries of the type considered here upto some critical value of the vorticity $\omega$. The existence of symmetrical Prandtl-Batchelor flows with two counter-rotating eddies remains an open question, and the possibility of generalizing the method used here to such flows is presently under consideration.

\section{Mathematical Formulation}

The flow is sketched in figure 1 . In region $I$ the flow is irrotational and hence we may introduce the complex velocity potential $w(z)=\Phi+\mathrm{i} \Psi$, where $\Phi$ is the velocity potential, $\Psi$ is the stream function and $z=x+\mathrm{i} y$, with $x$ and $y$ as shown in figure 1 . As $z \rightarrow \infty$

$$
w(z) \rightarrow U z \mathrm{e}^{-\mathrm{i} \alpha}+\frac{\mathrm{i} \Gamma \log z}{2 \pi},
$$

$U, \alpha$ and $\Gamma$ being respectively the magnitude of free-stream velocity, the angle of attack with respect to plate $O A$ and the clockwise circulation induced at infinity by the requirement of finite velocity (Kutta condition) at $O$. There is a stagnation point of the exterior irrotational flow at $P$ on the plate $O A$, and it can be shown (Tanveer 1983 ) that there are no other stagnation points besides $P$ and $O$. The flow is assumed to separate tangentially at $A$ and to reattach tangentially at $B . A B O A$ is a streamline on which $\operatorname{Im} w=0$ without any loss of generality. In region II the stream function satisfies

and on the boundary $A O B A$

$$
\nabla^{2} \Psi=\omega
$$

$$
\Psi=0
$$

The location of the vortex sheet $A B$ is determined by the pressure condition

$$
\left|\frac{\mathrm{d} w}{\mathrm{~d} z}\right|^{2}-(\nabla \Psi)^{2}=q^{2}
$$




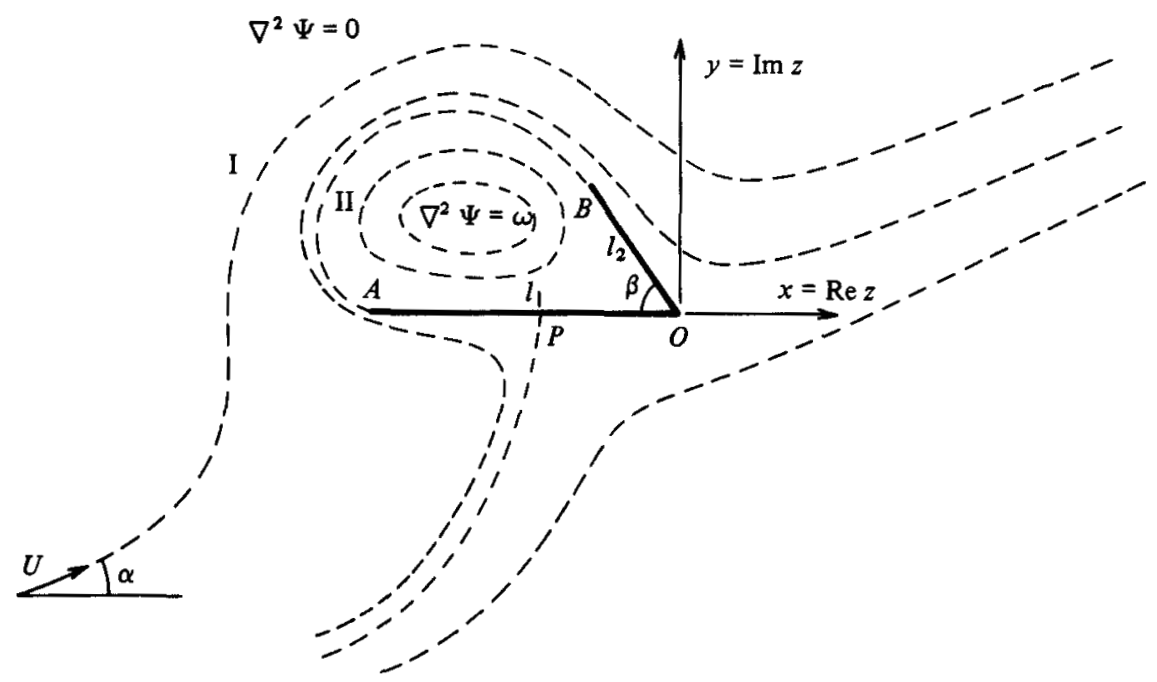

Figure 1. Sketch of flow under consideration. $A O B$ denotes the plate-flap combination, while the streamline joining $A$ to $B$ is the vortex-sheet location.

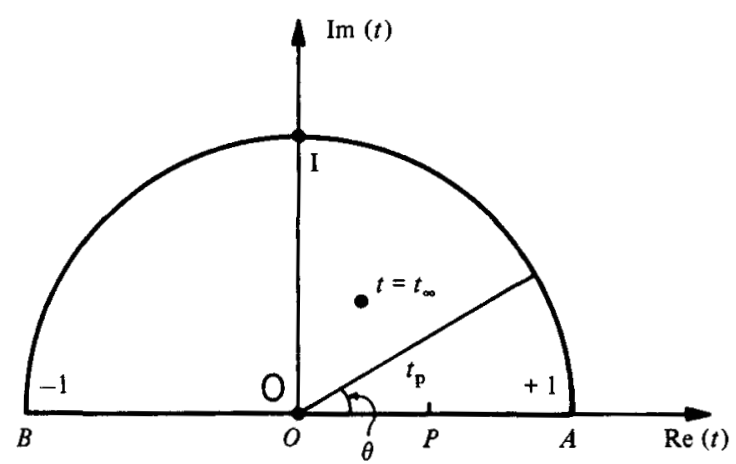

Figure 2. The $t$-plane.

where $\mathrm{d} w / \mathrm{d} z$ and $\nabla \Psi$ are evaluated on the vortex sheet from the outside and the inside respectively. $q^{2}$ is a constant which is twice the jump in the Bernoulli's constant across the boundary between regions I and II.

We consider the conformal map $t(z)$ of the exterior region $I$ into the interior of the unit semicircle as shown in figure 2. $A, B, O$ and $P$ in the $z$-plane correspond to 1 , $-1,0$ and $t_{\mathrm{p}}$ in the $t$-plane, where $t_{\mathrm{p}}$ is an unknown to be determined. We regard $\zeta=\mathrm{d} w / \mathrm{d} z$ as a function of $t$ and introduce the analytic function $\Omega(t)$ defined by

$$
\zeta=q t^{\beta / \pi} \frac{t_{\mathrm{p}}-t}{1-t t_{\mathrm{p}}} \mathrm{e}^{\Omega}
$$

where $0 \leqslant \arg (t) \leqslant \pi,-\pi \leqslant \arg \left(t_{\mathrm{p}}-t\right) \leqslant 0$ and $-\pi<\arg \left(1-t_{\mathrm{p}} t\right) \leqslant 0$ for $t$ in or on the unit semicirele. By considering the argument of both sides of (4) and assuming the continuity of $\zeta$, we conclude that $\operatorname{Im}(\Omega)=0$ on the real diameter of the unit semicircle. From the Schwarz reflection principle it follows that

$$
\Omega(t)=a_{0}+a_{1} t+a_{2} t^{2}+\ldots
$$


with $a_{0}, a_{1}, \ldots$ all real and $\Omega$ analytic in the interior of the unit circle. Now, introduce the conformal map, $T(t)$ defined by

$$
T=-\frac{1}{2}(t+1 / t) \text {. }
$$

If $T=T_{\infty}$ denotes the image of $z=\infty$, then the dipole and circulation present at infinity in the physical plane as in (1) implies that

$$
w=-\frac{M}{T-T_{\infty}}-\frac{\bar{M}}{T-\bar{T}_{\infty}}-\frac{\mathrm{i} \Gamma}{2 \pi}\left(\log \left(T-T_{\infty}\right)-\log \left(T-\bar{T}_{\infty}\right)\right),
$$

where $M$ is the complex magnitude of the dipole at $T=T_{\infty}$. Using (6) and (7) we easily conclude that

$\frac{\mathrm{d} w}{\mathrm{~d} t}=\frac{1-t^{2}}{2}\left[\frac{4 M t_{\infty}^{2}}{\left(t-t_{\infty}\right)^{2}\left(1-t t_{\infty}\right)^{2}}+\frac{4 \bar{M} t_{\infty}^{2}}{\left(t-\bar{t}_{\infty}\right)^{2}\left(1-t \bar{t}_{\infty}\right)^{2}}-\frac{\mathrm{i} \Gamma\left(t_{\infty}-\bar{t}_{\infty}\right)\left(1-t_{\infty} \bar{t}_{\infty}\right)}{\pi\left(t-t_{\infty}\right)\left(t-\bar{t}_{\infty}\right)\left(1-t t_{\infty}\right)\left(1-t \bar{t}_{\infty}\right)}\right]$,

where $t_{\infty}$ is the image of $z=\infty$ and is related to $T_{\infty}$ through (6). From (4) and (8) we obtain

$$
\begin{aligned}
& \frac{\mathrm{d} z}{\mathrm{~d} t}=\mathrm{e}^{-\Omega}\left(1-t^{2}\right) t^{-\beta / \pi} \frac{1-t t_{\mathrm{p}}}{2 q\left(t_{\mathrm{p}}-t\right)}\left[\frac{4 M t_{\infty}^{2}}{\left(t-t_{\infty}\right)^{2}\left(1-t t_{\infty}\right)^{2}}+\frac{4 \bar{M} \dot{t}_{\infty}^{2}}{\left(t-\bar{t}_{\infty}\right)^{2}\left(1-t \dot{t}_{\infty}\right)^{2}}\right. \\
& \left.-\frac{\mathrm{i} \Gamma\left(t_{\infty}-\bar{t}_{\infty}\right)\left(1-t_{\infty} \bar{t}_{\infty}\right)}{\pi\left(t-t_{\infty}\right)\left(t-\bar{t}_{\infty}\right)\left(1-t t_{\infty}\right)\left(1-t \bar{t}_{\infty}\right)}\right] .
\end{aligned}
$$

From the knowledge of the velocity at infinity, we have the condition

$$
U \mathrm{e}^{-\mathrm{i} \alpha}=q t_{\infty}^{\beta / \pi} \frac{t_{\mathrm{p}}-t_{\infty}}{1-t_{\mathrm{p}} t_{\infty}} \mathrm{e}^{\Omega\left(t_{\infty}\right)}
$$

Also, from the requirement that $\mathrm{d} z / \mathrm{d} t$ has no residue at $t=t_{\infty}$, which is necessary for the $z \rightarrow t$ mapping to be one-to-one, we have

$$
\frac{\mathrm{i} \Gamma}{2 \pi}=\frac{2 M t_{\infty}^{2}}{1-t_{\infty}^{2}}\left[-\frac{\beta}{\pi t_{\infty}}-\frac{t_{\mathrm{p}}}{1-t_{\mathrm{p}} t_{\infty}}+\frac{1}{t_{\mathrm{p}}-t_{\infty}}-\Omega^{\prime}\left(t_{\infty}\right)\right] .
$$

The geometric constraints are

$$
\begin{gathered}
\int_{0}^{1} \frac{\mathrm{d} z}{\mathrm{~d} t} \mathrm{~d} t=-l_{1}, \\
\int_{-1}^{0} \frac{\mathrm{d} z}{\mathrm{~d} t} \mathrm{~d} t=l_{2} \mathrm{e}^{-\mathrm{i} \beta},
\end{gathered}
$$

where $\mathrm{d} z / \mathrm{d} t$ is given by (9). Further, the stagnation points at $P$ and $O$ imply that $\mathrm{d} w / \mathrm{d} t=0$ at $t=t_{\mathrm{p}}$ and $t=0$. This means that

$$
\begin{gathered}
\frac{4 M t_{\infty}^{2}}{\left(t_{\mathrm{p}}-t_{\infty}\right)^{2}\left(1-t_{\mathrm{p}} t_{\infty}\right)^{2}}+\frac{4 \bar{M} t_{\infty}^{2}}{\left(t_{\mathrm{p}}-\bar{t}_{\infty}\right)^{2}\left(1-t_{\mathrm{p}} \bar{t}_{\infty}\right)^{2}}=\frac{\mathrm{i} \Gamma\left(t_{\infty}-\bar{t}_{\infty}\right)\left(1-t_{\infty} \bar{t}_{\infty}\right)}{\pi\left(t_{\mathrm{p}}-t_{\infty}\right)\left(t_{\mathrm{p}}-\bar{t}_{\infty}\right)\left(1-t_{\mathrm{p}} t_{\infty}\right)\left(1-t_{\mathrm{p}} \bar{t}_{\infty}\right)}, \\
4 M+4 \bar{M}=\frac{\mathrm{i} \Gamma\left(t_{\infty}-\bar{t}_{\infty}\right)\left(1-t_{\infty} \bar{t}_{\infty}\right)}{\pi t_{\infty} \bar{t}_{\infty}} .
\end{gathered}
$$

If $\Omega(t)$ were known inside the unit semicircle, then (10)-(15) would constitute eight real relations between the twelve real quantities $\Gamma, q, t_{\mathrm{p}}, \operatorname{Re} t_{\infty}, \operatorname{Im} t_{\infty}, \operatorname{Re} M, \operatorname{Im} M$, $l_{2}, l_{1}, \beta, U$ and $\alpha$. If we non-dimensionalize all our variables using $U$ and $l_{1}$, we are 
left with ten non-dimensional unknowns and eight real equations. There would then be a two-parameter family of solutions for the flow in the region I and its boundary. However, $\Omega(t)$ cannot be specified at will but has to be determined so that the interior flow in region II satisfies (3) on the vortex sheet. It will be seen later that the magnitude of the constant vorticity $\omega$, provided it is not too large, together with two of the parameters, say $\alpha$ and $\beta$, determine $\Omega(t)$ and all the other constants.

For the special case $\omega=0$, region II is stagnant and we have a free-streamline flow with the pressure condition (3) reducing to constancy of velocity on the free streamline. Comparing the magnitudes of both sides of (4) on the circumference of the unit semicircle, we deduce that $\operatorname{Re} \Omega=0$ on the circumference. Using the expansion (5), we conclude that $\Omega(t)=0$. For this case, the problem is completely determined by specification of the parameters $\alpha$ and $\beta$. Hurley (1959) obtained these solutions by a quite different procedure and presents the details of the two parameter family of solutions.

For the general case with $\omega \neq 0$, the determination of $\Omega(t)$ is more complicated, since the velocity in region II has to be taken into account in the pressure condition (3). For a vortex-sheet location corresponding to $t=\mathrm{e}^{\mathrm{i} \theta}$ for $\theta \in[0, \pi],(3)-(5)$ imply

$$
a_{0}+a_{1} \cos \theta+a_{2} \cos 2 \theta+\ldots=\frac{1}{2} \ln \left(1+(\nabla \Psi)^{2} / q^{2}\right) .
$$

The problem now reduces to determining the right-hand side of (16) and its Fourier cosine expansion with respect to $\theta$ in terms of $a_{0}, a_{1}, \ldots$ and the parameters. In $\S 3$ we describe how the inner velocity and hence the right-hand side of $(16)$ is determined for given $a_{0}, a_{1}, \ldots$ and $\Gamma, q, t_{\mathrm{p}}, t_{\infty}, M$ and $l_{2}$.

\section{Determination of the velocity in region II on the vortex sheet}

For given $a_{0}, a_{1}, \ldots$ and $\Gamma, q, t_{\mathrm{p}}, t_{\infty}, M, l_{2}$, we integrate (9), using $z(1)=-l_{1}$, to determine $z\left(\mathrm{e}^{\mathrm{i} \theta}\right)$, the location of the vortex sheet, and therefore the boundary of region II. We are then left with the problem of computing the velocity $|\nabla \Psi|$ on the free streamline, where $\Psi$ satisfies $(2 a, b)$. Since determination of the velocity on the vortex-sheet boundary of region II by the usual finite-difference or finite-element schemes in the physical plane is likely to be inaccurate in view of the infinite curvature expected at $A$ and $B$, we employ conformal mapping of region II into a more suitable region. It is convenient to separate $\Psi$ into a particular solution $\Psi_{\mathrm{p}}$ and a harmonic function $\Psi_{\mathrm{h}}$. Since $\omega$ is a constant, this can be easily done. $\Psi_{\mathrm{h}}$ remains a harmonic function in the conformally transformed plane. Then the problem is reduced to determining the normal derivative of a harmonic function from given boundary data in a 'nice' domain. For the purpose of mapping, we introduce the complex variable $z_{\mathrm{i}}=-x+\mathrm{i} y$ defined in region II of the physical plane. $\uparrow$ It is convenient to take the particular solution $\Psi_{\mathrm{p}}$ defined by

$$
\omega^{-1} \Psi_{\mathrm{p}}=\frac{1}{2}\left(\operatorname{Im} z_{\mathrm{i}}\right)^{2}-\frac{1}{2}\left(\operatorname{Im} z_{\mathrm{i}}\right)\left(\operatorname{Re} z_{\mathrm{i}}\right) \tan \beta
$$

since this vanishes on the solid boundaries. The harmonic function $\Psi_{\mathrm{h}}$ in the decomposition $\Psi=\Psi_{\mathrm{h}}+\Psi_{\mathrm{p}}$ satisfies $\Psi_{\mathrm{h}}=-\Psi_{\mathrm{p}}$ on the boundary

Consider the conformal map $Q\left(z_{1}\right)$ that maps the $z_{\mathrm{i}}$ plane interior of the boundaries $A O B A$ into the unit semicircle (figure 3 ), such that $B$ is mapped to $-1, A$ is mapped to +1 and $O$ to $Q_{0}$ on the real axis. The value of $Q_{0}$ is fixed automatically in the

$\dagger$ This unconventional choice of complex variable allows region II to be mapped to the interior of the semicircle of figure 3 . 

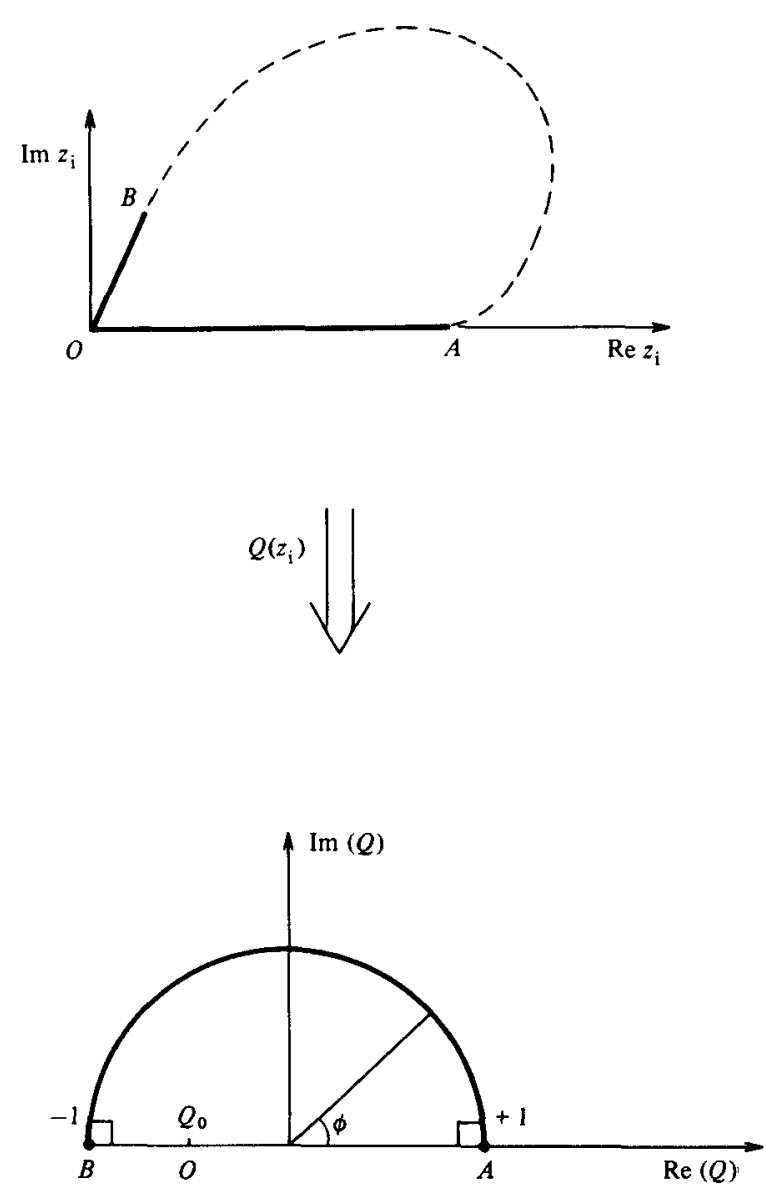

Figure 3. The $Q$-plane.

numerical conformal mapping procedure to be described later. The vortex sheet $A B$ is mapped to the semicircular boundary. The details of the mapping $z_{1}(Q)$ will be discussed in $\S 4$. We now consider $\Psi_{\mathrm{h}}$ as a harmonic function in the $Q$-plane which satisfies boundary conditions as follows:

$$
\Psi_{\mathbf{h}}\left(\mathrm{e}^{\mathrm{i} \phi}\right)=-\Psi_{\mathrm{p}}\left(z_{\mathbf{i}}\left(\mathrm{e}^{\mathrm{i} \phi}\right)\right)
$$

where $\phi \in[0, \pi]$ and $Q=\rho \mathrm{e}^{\mathrm{i} \phi}$ in the $Q$-plane. From $(17), \Psi_{\mathrm{p}}$ is zero on the real diameter, and so for $Q \in[-1,1]$ we have

$$
\Psi_{\mathrm{h}}(Q)=0 .
$$

Using the Schwarz reflection principle, we extend the domain of existence of $\Psi_{\mathrm{h}}$ to the entire unit $Q$-circle with boundary condition (18) applied to $\phi \in[0, \pi]$, while

$$
\Psi_{\mathrm{h}}\left(\mathrm{e}^{\mathrm{i} \phi}\right)=-\Psi_{\mathrm{h}}\left(\mathrm{e}^{-\mathrm{i} \phi}\right)
$$

extends the boundary data to the lower half segment of the circumference where $\phi \in[-\pi, 0]$. On the vortex sheet, the velocity $|\nabla \Psi|$ equals the normal derivative of $\Psi$ in the $z_{\mathrm{i}}$ plane. Now, the outward normal direction on the vortex sheet in the $z_{\mathrm{i}}$ plane corresponds to the radially outward direction on the circumference of the unit 
semicircle in the $Q$-plane. Therefore on $Q=\mathrm{e}^{\mathrm{i} \phi}$ the magnitude of the velocity is given by

$$
\begin{aligned}
|\nabla \Psi|=\left|\frac{\mathrm{d} Q}{\mathrm{~d} z_{\mathrm{i}}}\right|\left(\frac{\partial \Psi_{\mathrm{p}}}{\partial \rho}+\frac{\partial \Psi_{\mathrm{h}}}{\partial \rho}\right)=\operatorname{Im} z_{\mathrm{i}} & \frac{\operatorname{Im}\left(Q \frac{\mathrm{d} z_{\mathrm{i}}}{\mathrm{d} Q}\right)}{\left|\frac{\mathrm{d} z_{\mathrm{i}}}{\mathrm{d} Q}\right|}-\frac{1}{2} \tan \beta \frac{\operatorname{Re}\left(Q \frac{\mathrm{d} z_{\mathrm{i}}}{\mathrm{d} Q}\right)}{\left|\frac{\mathrm{d} z_{\mathrm{i}}}{\mathrm{d} Q}\right|} \operatorname{Im} z_{\mathrm{i}} \\
& +\left|\frac{\mathrm{d} z_{\mathrm{i}}}{\mathrm{d} Q}\right|^{-1} \frac{\partial \Psi_{\mathrm{h}}}{\partial \rho}-\frac{1}{2} \tan \beta \operatorname{Re} z_{\mathrm{i}} \frac{\operatorname{Im}\left(Q \frac{\mathrm{d} z_{\mathrm{i}}}{\mathrm{d} Q}\right)}{\left|\frac{\mathrm{d} z_{\mathrm{i}}}{\mathrm{d} Q}\right|} .
\end{aligned}
$$

Now (18) and (20) determine the harmonic function $\Psi_{\mathrm{h}}$ on the boundary of the unit circle from which the Fourier coefficients of the following expansion are determined:

$$
\Psi_{\mathrm{h}}\left(\mathrm{e}^{\mathrm{i} \phi}\right)=\sum_{-\infty}^{+\infty} \psi_{k} \mathrm{e}^{\mathrm{i} k \phi}
$$

It is easily seen that the normal derivative

$$
\frac{\partial \Psi_{\mathrm{h}}}{\partial \rho}\left(\mathrm{e}^{\mathrm{i} \phi}\right)=\sum_{-\infty}^{+\infty}|k| \psi_{k} \mathrm{e}^{\mathrm{i} k \phi}
$$

Using $z_{\mathrm{i}}(Q)$ and $\left|\mathrm{d} z_{\mathrm{j}} / \mathrm{d} Q\right|$ as determined in $\S 4,(23)$ provides a complete determination of $|\nabla \Psi|$ in (21) in terms of $\phi$. The apparent singularities at $\phi=0$ and $\pi$ are actually removable since $\Psi$ and therefore $\partial \Psi / \partial \rho$ are odd $2 \pi$-periodic functions of $\phi$ and hence vanish at 0 and $\pi$. It is to be noted that in (16) we need $|(\nabla \Psi)|^{2}$ as a function of $\theta$ and not $\phi$, as given by (21). However, for each $\theta,(9)$ locates $(x(\theta, y(\theta))$ on the physical vortex sheet and therefore the corresponding position in the $z_{1}$ plane. The conformal map $Q\left(z_{\mathrm{i}}\right)$ provides the relation $\phi\left(z_{\mathrm{i}}(\theta)\right)$ needed to determine the velocity as a function of $\theta$. The details of this correspondence and its usage are discussed in $\S 5$.

\section{Mapping into a semicircle in the $Q$-plane}

As mentioned before, we wish to find $Q\left(z_{\mathrm{i}}\right)$ that maps the $z_{\mathrm{i}}$ plane into a semicircle with $A$ mapped to $+1, B$ to -1 and $O$ to some suitable point $Q_{0}$ on the real diameter of the $Q$-plane. Initially, we carry out a series of explicit transformations starting with the $z_{\mathbf{i}}$ plane and finally ending up with a geometry that is close to a semicircle. With choice of appropriate branches, the transformations are

$$
\begin{aligned}
W_{1}\left(z_{\mathrm{i}}\right) & =\left(z_{\mathrm{i}}^{\pi / \beta}-s_{1}\right) / E, \\
W_{2}\left(W_{1}\right) & =-1-\frac{2 \mathrm{i}}{\pi} \log \left(-W_{1}+\mathrm{i}\left(1-W_{1}^{2}\right)^{\frac{1}{2}}\right), \\
W_{3}\left(W_{2}\right) & =\frac{\left(r \sin \frac{1}{2} \delta+W_{2}\right)^{\pi / \delta}-\left(r \sin \frac{1}{2} \delta-W_{2}\right)^{\pi / \delta}}{\left(r \sin \frac{1}{2} \delta+W_{2}\right)^{\pi / \delta}+\left(r \sin \frac{1}{2} \delta-W_{2}\right)^{\pi / \delta}},
\end{aligned}
$$

where $s_{1}=\frac{1}{2}\left(1-l_{2}^{\pi / \beta}\right)$ and $E=\frac{1}{2}\left(1+l_{2}^{\pi / \beta}\right) . r$ is chosen to be the radius of the circular arc shown in dotted line in figure $4(b)$, which together with the real axis encloses our region of interest in the $W_{2}$ plane. The angle between the circular arc and the real axis is $\frac{1}{2} \delta$. Figures $4(a-c)$ show the approximate shapes of the region of interest after each of the transforms (24)-(26). The transformation (26) converts the circular are and the real axis of figure $4(b)$ into a unit semicircular boundary If $r \sin \frac{1}{2} \delta$ is chosen 
(a)
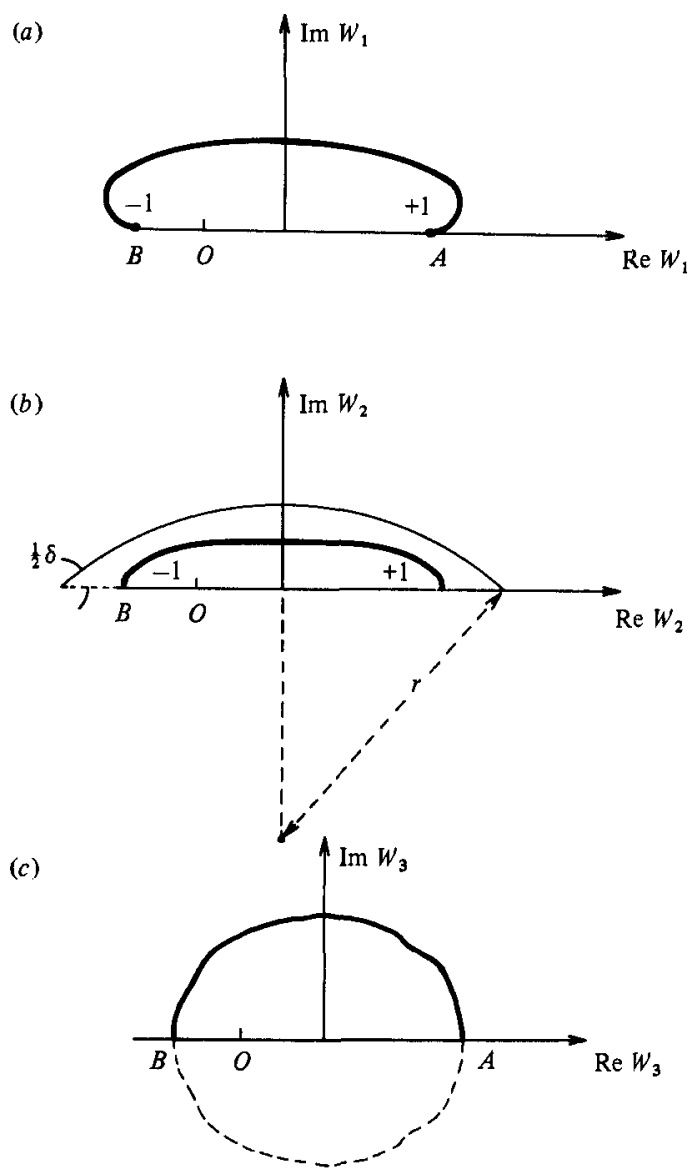

Figure 4. $W_{1}, W_{2}$ and $W_{3}$ planes shown in $(a),(b)$ and $(c)$ respectively. $r$ denotes the radius of the circular arc that, together with real axis, encloses the geometry of interest in $(b) . \frac{1}{2} \delta$ is the angle between the circular arc and the real axis.

close to 1 , the transformation (26) gets rid of the large curvature of the boundary of the region of interest in the $W_{2}$ plane near the real axis. The choice of $r$ and $\delta$ is made so as to make the shape of the boundary in the $W_{3}$ plane as nearly semicircular as possible.

Consider now the function $W_{3}(Q)$ that maps a semicircle into this nearly semicircular region with the origin 0 of the $Q$-plane mapped to 0 in the $W_{3}$ plane, -1 to -1 and +1 to +1 . We realize that $W_{3}(Q)$ also maps the unit circle about the origin in the $Q$-plane into the nearly circular geometry of the $W_{3}$ plane formed by extending the original nearly semicircular region through reflection on the real axis. Henceforth, the nearly circular region of the $W_{3}$ plane will be called the extended $W_{3}$ region. If $W_{3}=R_{w} \mathrm{e}^{\mathrm{i} \nu}$ and $Q=\mathrm{e}^{\mathrm{i} \phi},-\pi \leqslant \nu, \phi \leqslant \pi$, characterize corresponding points on the boundary of the extended $W_{3}$ region and the unit circle respectively, then the integral equation satisfied by $\nu(\phi)$ is

$$
\nu(\phi)-\phi=\frac{1}{2 \pi} \int_{-\pi}^{\pi} \cot \left(\frac{\phi-\phi^{\prime}}{2}\right) \log \left(R_{w}\left(\nu\left(\phi^{\prime}\right)\right)\right) \mathrm{d} \phi^{\prime},
$$


where the integral is in the principal-value sense. This equation, commonly known as Theodersen's integral equation, is very easily solved numerically in Fourier space by an iterative technique as discussed by Henrici (1979). It requires two Fourier transforms and one $R_{w}$ evaluation per iteration. Once the iterations converge, we obtain the complex Fourier series for $\log \left(R_{w}(\nu(\phi))\right)$ which allows us to find the coefficients of the Fourier cosine series in $\phi$ as well, since it is an even periodic function. Thus we can write

from which it follows that

$$
\log \left(R_{w}(\nu(\phi))\right)=\sum_{0}^{\infty} b_{n} \cos n \phi,
$$

$$
\log \frac{W_{3}}{Q}=\sum_{0}^{\infty} b_{n} Q^{n}
$$

since the left-hand side of (28) is the real part of the analytic function $\log \left(W_{3} / Q\right)$ evaluated at the boundary of the unit circle. Thus we have arrived at a power-series expression to describe the mapping function $W_{3}(Q)$ that maps the circle in the $Q$ plane to the extended $W_{3}$ region and therefore the semicircle into the original nearsemicircular region of the $W_{3}$ plane. Inverting the relations in (24)-(26) and using (29), we arrive at $z_{\mathfrak{i}}(Q)$, from which $Q\left(z_{\mathrm{j}}\right)$ is known in principle. The velocity is therefore determined from the inside of the vortex sheet for a given vortex-sheet location. Equating the coefficients of $\cos n \theta$ in (16), the coefficients $a_{0}, a_{1}, \ldots$ are determined.

Here, we justify the mapping of region II into a semicircle to determine the velocity on the vortex sheet. The infinite curvature of the separating streamline and infinite velocity gradient on the plate (or the flap) at $A$ (or $B$ ), as will be seen in $\$ 7$, leads to numerical inaccuracies if a direct scheme (such as finite differences) is used to calculate the flow in region II on the vortex-sheet boundary. The commonly used technique of conformal mapping into a circle does not get rid of the problem either, because the boundary data assumed by the harmonic decomposition $\Psi_{h}$, as introduced in $\S 3$, is not a smooth function of the angle on the circle. Mapping into a semicircle with the separation and reattachment points at the two ends of the real diameter, as we have done, gets rid of the problem of accurately calculating the velocity close to the separation and reattachment points. The infinite velocity gradients in the physical plane present no obstacles, since the numerically calculated $\Psi_{\mathrm{h}}, \Psi_{\mathrm{p}}$ and therefore the velocity seem to be smooth functions of $\phi$ on the $Q$-circle. Further, the numerical calculations suggest strongly that the velocity on the boundary is a smooth function of $\theta$ as well in the interval $[0, \pi]$. Smoothness of the interior velocity as a function of $\theta$ implies that $\Omega$, calculated by using (16), is analytic at the boundary of the unit circle. This smoothness of $\Psi$ and the velocity and the analyticity of $\Omega$ can be expected from further plausibility arguments (Tanveer 1983). Thus the numerical calculation of velocity in region II is easily facilitated even in the neighbourhood of $A$ and $B$ by using the conformal mapping into the $Q$-plane as described.

\section{Numerical procedure}

We use an iterative scheme to find solutions to our equations. We start with an initial guess of $N$ coefficients $a_{0}, a_{1}, \ldots, a_{N-1}$ and obtain an approximate $\Omega(t)$ by truncating (5). For small $\omega$ the guess for $\Omega$ and hence these $N$ coefficients is zero. For 
bigger values of $\omega$ we use values of the coefficients obtained from the converged solution for slightly smaller $\omega$. In the following we describe the procedure used to find the $(n+1)$ th iterate given the $n$th iterates $a_{0}^{n}, a_{1}^{n}, \ldots, a_{N-1}^{n}$ and therefore $\Omega^{n}(t)$.

We use (10)-(15) to determine $\Gamma, l_{2}, q, M, t_{\infty}$ and $t_{\mathrm{p}}$ consistent with $\Omega=\Omega^{n}$, where $U, l_{1}, \alpha$ and $\beta$ are considered known and fixed. Newton iteration is used for that purpose. Equation (9) then determines $z(\theta)=x(\theta)+i y(\theta)$ and hence $z_{i}(\theta)=-x(\theta)+\mathrm{i} y(\theta)$ on the vortex sheet for $N_{1}$ points where $\theta_{k}=(k-1) \pi /\left(N_{1}-1\right)$, $k=1,2, \ldots, N_{1}$, and $N_{1}$ is chosen of the form $N_{1}=1+2 N l$, where $l$ is any positive integer. A subset of these $\theta$-values of the form $\theta_{k_{j}}=(((2 j-1) l+1)-1) \pi /\left(N_{1}-1\right)$ obtained for $k=(2 j-1) l+1$ for $j=1,2, \ldots, N$ are the $\theta$-values at which velocities will be calculated and (16) used to find the $N$ coefficients $a_{j}$. A little simplification shows that $\theta_{k j}=(j-1) \pi / N+\pi / 2 N$ and are therefore points at which the $N$ calculated velocities can be fast-Fourier-transformed to obtain the Fourier cosine series of (16). We avoid calculating velocities at $\theta=0$ and $\pi$, where (21) has numerically awkward removable singularities. Now, we find the images $R_{w}\left(\nu_{k}\right) \mathrm{e}^{\mathrm{i} \nu_{k}}$ in the $W_{3}$ plane for these $N_{3}$ points in the $z_{i}$ plane, using (24)-(26). The $v$-values in the $W_{3}$ plane image of the subset of $z_{i}$ points characterized by the angles $\theta_{k j}$ are denoted by $v_{k j}$. Reflection on the real axis provides us with a set of $N_{1}-2$ new points on the near-circular boundary of the extended $W_{3}$ region. All together, we then have $2 N_{1}-2$ points at which $R_{w}\left(\nu_{k}\right)$, $v_{k}$ are known. For large enough $N_{1}$ this provides a very accurate description of the function $R_{w}(\nu)$ for arbitrary $\nu$ through cubic spline interpolation. We then take $N_{3}$ points on the unit circle of the extended $Q$-plane, evenly spaced in the angular variable $\phi$, and carry out the process of solving Theodersen's integral equation (27) exactly as described by Henrici (1979). In the process, we obtain the first $\frac{1}{2} N_{3} b_{n}$ coefficients in (28). The $b_{n}$ coefficients define $W_{3}(Q)$ in (29). We then start with $N_{2}$ uniformly spaced points on the circumference of the unit $Q$-circle with $Q_{m}=\mathrm{e}^{2 \pi \mathrm{i}(m-1) / N_{2}}$ for $m=$ $1,2, \ldots, N_{2}$. We calculate $W_{3}\left(Q_{m}\right), z_{i}\left(W_{3}\left(Q_{m}\right)\right)$ using $(24)-(26)$ and $(29)$ at those points. Using (17), (18) and (20) we calculate $\Psi_{\mathrm{h}}$ at those boundary points and use them in (22) and (23) to find $\partial \Psi_{\mathrm{h}} / \partial \rho$ at $N_{2}$ points. At the $\frac{1}{2} N_{2}-1$ points that lie entirely in the upper-half $Q$ semicircular boundary we calculate $\mathrm{d} z_{\mathrm{i}} / \mathrm{d} Q$ using (24)-(26) and (29). These, together with values of other terms in (21) already calculated, provide the velocities at the $\frac{1}{2} N_{2}-1$ points on the upper-half semicircle. If $\nu_{m}, m=1,2, \ldots, \frac{1}{2} N_{2}-1$, denote the angular positions of the images of those $Q$-points in the $W_{3}$ plane, we use these to interpolate velocities at the $N$ points $\nu_{k_{j}}$ through cubic splines. Because of the correspondence of $\nu_{k_{j}}$ with $N$ uniformly spaced-out points in the $\theta$-variable as discussed earlier, we arrive at the velocities from the inner side of the vortex sheet at the physical $z$-locations corresponding to $N$ equispaced points in the $\theta$-variable. This is exactly as desired, since (16) allows calculation of the $(n+1)$ th iterate for $a_{0}, a_{1}, \ldots, a_{N-1}$ by fast Fourier transforms as in Henrici (1979).

Thus we have a full description of the iteration scheme. It may be noted that the use of spline interpolation in the variable $v$ is suitable because, as argued in $\$ 4$, the velocity is a smooth function of $\phi$ and $\theta$.

\section{Numerical results and discussion}

The object of the present work is to demonstrate the existence of Prandtl-Batchelor flows, and we only present results here for the case $\beta=1.5 \mathrm{rad}, \alpha=0.3 \mathrm{rad}$, although solutions do exist for a range of $\beta$ and $\alpha$. Future calculations for other values of parameters would be dictated by practical interest and necessity. It appears that for these values $\beta$ and $\alpha$, solutions exist when the vorticity in region II is in the ranre 


\begin{tabular}{|c|c|c|c|c|}
\hline & $\omega l_{1} / U=0$ & $\omega l_{1} / U=4$ & $\omega l_{1} / U=8$ & $\omega l_{1} / U=10$ \\
\hline$\Gamma / U l_{1}$ & 3.774983 & 3.832852 & 4.040451 & 4.243160 \\
\hline$q / U$ & 2.738574 & 2.669800 & 2.400910 & 2.097837 \\
\hline $\operatorname{Re} t_{\infty}$ & 0.1756735 & 0.1733073 & 0.1652458 & 0.1578012 \\
\hline $\operatorname{Im} t_{\infty}$ & 0.4323535 & 0.4343168 & 0.4416190 & 0.4491662 \\
\hline $\operatorname{Re} M / q l_{1}$ & -0.1703347 & -0.1772941 & -0.2068618 & -0.2466787 \\
\hline $\operatorname{Im} M / q l_{1}$ & -0.1449536 & -0.1491897 & -0.1677067 & -0.1937230 \\
\hline$t_{\mathrm{p}}$ & 0.4187071 & 0.4180502 & 0.4172719 & 0.4190171 \\
\hline$l_{2}$ & 0.3445046 & 0.3522005 & 0.3791436 & 0.4044527 \\
\hline$a_{0}$ & 0 & 0.0183422 & 0.0966727 & 0.2006016 \\
\hline$a_{1}$ & 0 & -0.0039603 & -0.0241468 & -0.0539812 \\
\hline$a_{2}$ & 0 & -0.0167881 & -0.0776793 & -0.1388259 \\
\hline$a_{3}$ & 0 & -0.0024419 & -0.0125488 & -0.0260786 \\
\hline$a_{4}$ & 0 & 0.0045678 & 0.0162926 & 0.0208911 \\
\hline$a_{5}$ & 0 & 0.0023665 & 0.0099788 & 0.0152292 \\
\hline$a_{6}$ & 0 & -0.0004640 & 0.0001409 & 0.0022860 \\
\hline$a_{7}$ & 0 & -0.0008054 & -0.0021805 & -0.0017057 \\
\hline$a_{8}$ & 0 & -0.0002034 & -0.0011002 & -0.0012923 \\
\hline$a_{9}$ & 0 & 0.0001294 & -0.0000245 & -0.0002766 \\
\hline$a_{10}$ & 0 & 0.0000974 & 0.0001879 & -0.0000072 \\
\hline$a_{11}$ & 0 & 0.0000102 & 0.0001080 & 0.0000350 \\
\hline$a_{12}$ & 0 & -0.0000222 & 0.0000034 & -0.0000109 \\
\hline$a_{13}$ & 0 & -0.0000107 & -0.0000121 & 0.0000058 \\
\hline$a_{14}$ & 0 & -0.0000009 & -0.0000151 & -0.0000063 \\
\hline$a_{15}$ & 0 & 0.0000027 & -0.0000030 & 0.0000015 \\
\hline$a_{16}$ & 0 & 0.0000004 & -0.0000043 & -0.0000088 \\
\hline$a_{17}$ & 0 & -0.0000001 & 0.0000002 & -0.0000027 \\
\hline$a_{18}$ & 0 & -0.0000008 & -0.0000023 & -0.0000066 \\
\hline$a_{19}$ & 0 & -0.0000001 & 0.0000000 & -0.0000014 \\
\hline$a_{20}$ & 0 & -0.0000003 & -0.0000018 & -0.0000041 \\
\hline$a_{21}$ & 0 & 0.0000000 & -0.0000002 & -0.0000008 \\
\hline$a_{22}$ & 0 & -0.0000002 & -0.0000014 & -0.0000029 \\
\hline$a_{23}$ & 0 & 0.0000000 & -0.0000001 & -0.0000007 \\
\hline$a_{24}$ & 0 & -0.0000002 & -0.0000010 & -0.0000021 \\
\hline$a_{25}$ & 0 & 0.0000000 & -0.0000001 & -0.0000005 \\
\hline$a_{28}$ & 0 & -0.0000001 & -0.0000008 & -0.0000016 \\
\hline$a_{27}$ & 0 & 0.0000000 & -0.0000001 & -0.0000004 \\
\hline$a_{28}$ & 0 & -0.0000001 & -0.0000006 & -0.0000013 \\
\hline$a_{29}$ & 0 & 0.0000000 & -0.0000001 & -0.0000003 \\
\hline$a_{30}$ & 0 & -0.0000001 & -0.0000004 & -0.0000010 \\
\hline$a_{31}$ & 0 & -0.0000001 & -0.0000001 & -0.0000002 \\
\hline$a_{32}$ & 0 & -0.0000001 & -0.0000003 & -0.0000008 \\
\hline$a_{33}$ & 0 & 0.0000000 & -0.0000001 & -0.0000001 \\
\hline$a_{34}$ & 0 & 0.0000000 & -0.0000002 & -0.0000006 \\
\hline$a_{35}$ & 0 & 0.0000000 & 0.0000000 & -0.0000001 \\
\hline$a_{36}$ & 0 & 0.0000000 & -0.0000002 & -0.0000004 \\
\hline$a_{37}$ & 0 & 0.0000000 & 0.0000000 & -0.0000001 \\
\hline$a_{38}$ & 0 & 0.0000000 & -0.0000002 & -0.0000003 \\
\hline$a_{39}$ & 0 & 0.0000000 & 0.0000000 & -0.0000001 \\
\hline$a_{40}$ & 0 & 0.0000000 & -0.0000001 & -0.0000002 \\
\hline$a_{41}$ & $\mathbf{0}$ & 0.0000000 & 0.0000000 & 0.0000000 \\
\hline$a_{42}$ & 0 & 0.0000000 & 0.0000001 & -0.0000001 \\
\hline$a_{43}, a_{44}, a_{45}$ & 0 & 0.0000000 & 0.0000000 & 0.0000000 \\
\hline
\end{tabular}

TaBle 1. Values of quantities of interest for four different values of vorticity, each for $\alpha=0.3 \mathrm{rad}$ and $\beta=1.5 \mathrm{rad}$ 


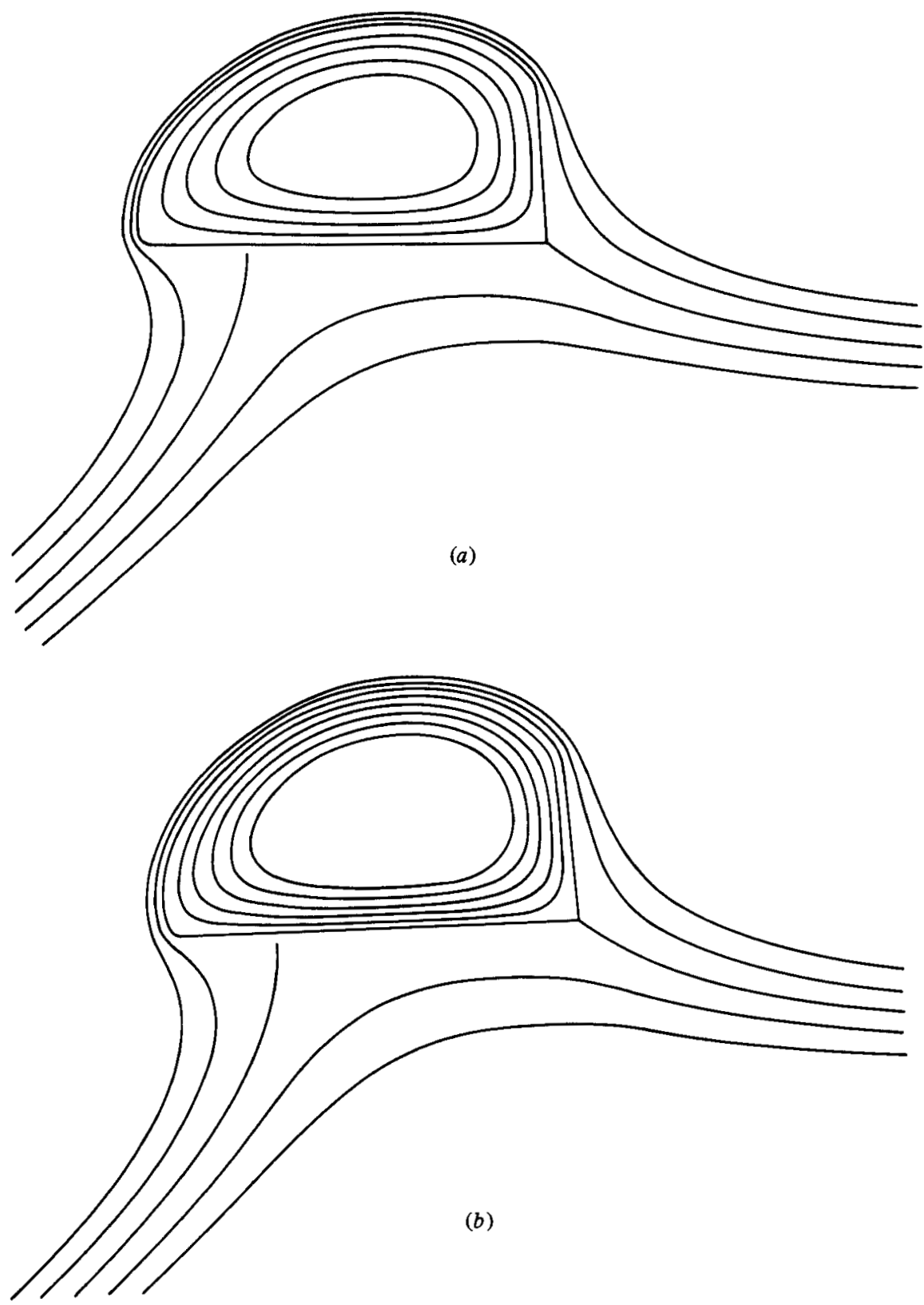

Fratre 5. Streamlines for: $(a) \omega l_{1} / U=8, \alpha=0.3 \mathrm{rad}, \beta=1.5 \mathrm{rad} ;(b) \omega l_{1} / U=10, \alpha=0.3 \mathrm{rad}$, $\beta=1.5 \mathrm{rad}$. Streamlines are drawn at intervals of 0.04 for $\Psi / U l_{1}$.

$0 \leqslant w l_{1} / U \leqslant 10$. The iteration procedure described in $\S 5$ was used to solve for $\Omega(t)$. When the successive calculated values of the coefficients $a_{0}, a_{1}, \ldots, a_{N-1}$ and other constants were within $10^{-8}$ of each other, convergence was assumed. Initially, in our calculations, we used $N_{1}=385, N=64, N_{2}=4096$ and $N_{3}=512$. Changing each of $N_{1}, N, N_{2}$ and $N_{3}$ made little difference in the converged numerical values. For instance when $N_{1}=385, N=48, N_{2}=2048$ and $N_{3}=512$, the calculated values of the constants $\Gamma / l_{1} U, q / U$, etc. and the values that $\Omega$ assumed on a host of different 
points on the $t$-circle were identical with the originally obtained values upto seven significant figures. Thus we conclude that the calculations have a seven-digit accuracy.

The values of different quantities obtained from calculation are presented in table 1 for four different values of vorticity $\omega$. The streamlines are shown in figures $5(a)$ and $(b)$ for the cases $\omega l_{1} / U=8$ and $\omega l_{1} / U=10$. The results suggest that the vortex sheet moves outwards, and hence the value of $l_{2}$ needed to assure reattachment at $B$ increases with $\omega l_{1} / U$. The extensive use of fast Fourier transforms and spline interpolation avoided the use of any integrations in the calculation of the velocity in region II, and therefore we believe that the method is comparatively very efficient compared to the integro-differential equation techniques used by others who tried calculation of these types of flows. For each value of $\omega l_{1} / U$, the entire calculation took between 10 and 20 min of CPU time on a VAX 11/750. We obtained numerical convergence in our iteration scheme upto $\omega l_{1} / U=10$. For larger values the rate of convergence slowed down considerably, suggesting that there was a critical value of $\omega l_{1} / U$ above 10 for which there will be no convergence in the iteration scheme. We are unable to explain the significance of a critical value of $\omega$. For $\omega l_{1} / U=8$ the method needed 8 iterations to produce a seven-figure-accurate result. A different set of values for $N_{1}, N, N_{2}$ and $N_{3}$ made small differences in the convergence rates, provided that those integer values were large enough.

\section{Nature of singularities}

In this section we point out the nature of the singularities of the flow field at the separation and reattachment points $A$ and $B$. First, we consider the exterior flow field. If we expand (4) around $t=1$, taking $\Omega$ to be analytic as was justified earlier, we obtain

$$
\frac{\mathrm{d} w}{\mathrm{~d} z}=k_{1}+k_{2}(t-1)+k_{3}(t-1)^{2}+\ldots
$$

where $k_{1}, k_{2}$ and $k_{3}$ are all real constants. A similar expansion is valid at $t=-1$, with the factor $t-1$ replaced by $t+1$, and the constants $k_{1}, k_{2}$ and $k_{3}$ are each proportional to $\mathrm{e}^{\mathrm{i} \beta}$. If we expand (9) around $t=1$ and integrate under the assumption that the term within the square brackets in (9) is non-zero (justified in Tanveer 1983), we find that

$$
z+l_{1}=p_{1}(t-1)^{2}+p_{2}(t-1)^{3}+\ldots,
$$

where $p_{1}$ and $p_{2}$ are real. A similar expression is valid at $t=-1$, with the factor $t-1$ replaced by $t+1$, the constants $p_{1}$ and $p_{2}$ are proportional to $\mathrm{e}^{-\mathrm{i} \beta}$ and the term $l_{1}$ is replaced by $l_{2} \mathrm{e}^{-\mathrm{i} \beta}$. Evaluating the real and imaginary parts of (31) for $t=\mathrm{e}^{\mathrm{i} \theta}$, and eliminating $\theta$, we find that in the neighbourhood of $A$ on the separating streamline

$$
y=y_{1}\left(l_{1}+x\right)^{\frac{3}{2}}+y_{2}\left(l_{1}+x\right)^{\frac{5}{2}}+\ldots,
$$

where $y_{1}$ and $y_{2}$ are real constants. A similar equation is valid at $B$, where $y$ and $l_{1}+x$ have to be replaced by the distances from $B$ in the direction perpendicular to and along the flap $O B$ respectively. From the numerically determined values, it was found that $y_{1}$ was non-zero at both $A$ and $B$ in the range of values of vorticity $\omega$ for which the calculations were made. Evaluating (30) and (31) with $t=\mathrm{e}^{\mathrm{i} \theta}$, it follows after some algebra that $|\mathrm{d} w / \mathrm{d} z|$. and hence the pressure, is a smooth function of the distance from $A$ along the separating streamline. The same smoothness is found at $B$. However, 
from (30), (31) and similar equations in the neighbourhood of $B$, it follows that the pressure gradient tends to infinity as $A$ (or $B$ ) is approached along the plate (or the flap). From the numerical values of the coefficients of $\Omega$ and the other constants, it was found that the pressure gradient was favourable on the plate at $A$ and unfavourable on top of the flap at $B$.

Now we consider the flow-field singularities in region II at $A$ and $B$. From (22) and the fact that $\Psi_{\mathrm{h}}$ is a real odd $2 \pi$-periodic function of $\phi$, it follows that at an anterior point $\rho \mathrm{e}^{\mathrm{i} \phi}$ in the $Q$-plane

$$
\Psi_{\mathrm{h}}=\sum_{1}^{\infty} c_{k} \rho^{k} \sin k \phi
$$

where the $c_{k}$ are all real and related to the coefficients $\psi_{k}$ in (22). From (17), (33) and from the expansion of $\left|\mathrm{d} z_{\mathbf{i}} / \mathrm{d} Q\right|$ and $z_{\mathbf{i}}(Q)$ near $Q=1$ and -1 , obtained from (24)-(26) and (29), we find that the magnitude of the velocity on the plate $O A$ or the flap $O B$ in the neighbourhood of $A$ or $B$ is given by

$$
|\nabla \Psi|=V_{0}+V_{1} s^{\frac{1}{2}}+\ldots,
$$

where $V_{0}$ and $V_{1}$ are constants and $s$ is the distance from $A$ or $B$, as the case may be. It was found from the numerical values of the coefficients and the constants in (22), (24)-(26) and (29) that in the range of $\omega$ for which calculations were made, $V_{1}$ was always positive at both $A$ and $B$, except for the case when $\omega=0$, for which the velocity in region II is identically zero. Thus, in region II, there is an adverse infinite pressure gradient on the plate at $A$ and an infinite favourable pressure gradient on the flap at $B$.

\section{Conclusion}

We have presented calculations of a so-called Prandtl-Batchelor flow for flow past a flat plate with a flap attached at its rear edge. This is to our knowledge the first such fully consistent calculation of a Prandtl-Batchelor flow past a physical body. For given angle of attack and angle between the plate and the flap, there exists a one-parameter family of such flows, depending on the assumed vorticity in the recirculating region of the flow. If the flow is the zero-viscosity limit of a Navier-Stokes solution, it is expected that only one such value of the vorticity will be consistent with the fitting of boundary layers. This question appears to be rather difficult and is not addressed this paper. Alternatively, the ratio $l_{2} / l_{1}$ over a certain range could be supposed to be given in addition to the angles $\alpha$ and $\beta$, and the value of $\omega$ could be regarded as determined by the requirement that the streamline that separates at $A$ reattaches at $B$.

Our method provides an accurate and reasonably efficient method of calculation of two dimensional Prandtl-Batchelor flows that incorporate possible singularities at the separation and reattachment points. The procedure is general and could possibly be applied to other two-dimensional geometries of interest.

This work was supported by the Office of Naval Research and Department of Energy, Office of Basic Energy Sciences (DE-AMO3-76SF00767). We also like to express gratitude to Dr Bengt Fornberg for some helpful discussions and useful suggestions. We also wish to thank the referees for some constructive criticism. 


\section{REFERENCES}

Batchelor, G. K. $1956 a$ On steady laminar flow with elosed streamlines at large Reynolds number. J. Fluid Mech. 1, 177-190.

Batchelor, G. K. $1956 b$ A proposal concerning laminar wakes behind bluff bodies at large Reynolds number. J. Fluid Mech. 1, 388-398.

DEEM, G. S. \& ZaBUsky, N. 1978 Stationary ' $V$-states', interactions, recurrence and breaking. In Solitons in Action (ed. K. Lonngren \& A. Scott). Academic.

Dzugaev, H. 1982 Solution of flow over a diaphragm in a two dimensional channel. Izv. Akad. Nauk SSSR, Mekh. Zhid. i Gaza, July-August, 134-137.

Henrici, P. 1979 The use of fast Fourier transform in computational complex analysis. Siam Rev. 21, 481-527.

Herwig, H. 1982 Die Anwendung der asymptotischen Theorie auf laminare Stromungen mit endlichen Ablosegebieten. Z. Flugwiss, Weltraumforsch. 6, 266-279.

Hurley, D. 1959 The use of boundary layer control to establish free streamline flows. Adv. Aero. Sci. 2, 662-708.

Prerrehumbert, R. T. 1980 A family of steady, translating vortex pairs with distributed vorticity. J. Fluid Mech. 99, 129-144.

SAdovskir, V.S. 1971 Vortex regions in a potential stream with a jump of Bernoulli's constant at the boundary. Prikl. Math. Mekh. 35, 773-779.

Saffman, P. G. 1981 Dynamics of vorticity. J. Fluid Mech. 106, 49-58.

Tanvenr, S. 1983 Topics in 2-d separated vortex flows. Ph.D. thesis, California Institute of Technology. 